\title{
Candidate vectors of horizontal transfer of BovB retrotransposon
}

\author{
Olga Skobel $^{1, *}$, Gleb Kosovsky ${ }^{1}$, and Valery Glazko ${ }^{1,2}$ \\ ${ }^{1}$ Scientific Research Institute of Fur-Bearing Animal Breeding and Rabbit Breeding named after V.A. \\ Afanas'ev, 140143, 6, Trudovaya, Moscow province, Ramensky region, Rodniki, Russian Federation \\ 2 Timiryazev Russian State Agrarian University-Moscow Agrarian Academy, 127550, 49, \\ Timiryazevskaya, Moscow, Russian Federation
}

\begin{abstract}
The horizontal transfer of retrotransposons has a significant impact on the regulatory system of a multicellular organism, but the biological basis of horizontal transfer has been sufficiently studied up to date. Earlier, we identified the conserved sequence of retrotransposons recombination products of the bovine chromosome 1 nucleotide sequence region. This conserved sequence has a high percent identity with LINE BovB, which is widely known as horizontal transfer participant. The current study analyzes the presence of the conserved sequence of retrotransposons recombination products of cattle in members of different taxonomic groups to detect potential vectors of horizontal transfer. It was shown that the conserved sequence with a high percent identity can be found in 43 members of different species, including eukaryotes, prokaryotes and viruses. The identified potential vectors of horizontal retrotransposon transfer associated with various diseases of farm animals are of particular interest. Such potential vectors are hemiparasites Babesia ovata and Babesia bigemina (pathogens causing babesiosis), bacterium Clostridium botulinum (the causative agent of botulism), Jaagsiekte sheep retrovirus (the causative agent of lung cancer in sheep). They all have regions with a high percent identity (not lower than 95\%) to the studied bovine conserved sequence. Thus, we identify new potential vectors of horizontal retrotransposon transfer as well as the possible influence of retrotransposons on regulatory networks affecting host protection from infectious diseases.
\end{abstract}

\section{Introduction}

The mobile genetic elements distribution, which is based on new microRNA forming, has a significant impact on regulatory network transformation of multicellular organisms including influencing the development of various diseases [1,2,3].

Difficulties in the description of multicellular organism regulatory networks are based on the high frequency of recombination of retrotransposons that make up a significant part of the eukaryotic genome [4]. Earlier we identified recombination products between the long interspersed nuclear element (LINE) and endogenous retrovirus (ERV) in the bovine

*Corresponding author: skobelolga@gmail.com 
chromosome 1 nucleotide sequence region of 13436028 in length [5]. It was found that 30 RTE-BovB/BTLTR1/RTE-BovB constructions are localized in the intronic regions of 12 structural genes and have a high percent identity (not lower than 78,32\%). These results allow identifying the conserved sequence of $266 \mathrm{bp}$ in length having percent identity not lower than $88.72 \%$ with all RTE-BovB/BTLTR1/RTE-BovB constructions. The studied conserved sequence is the part of LINE BovB (with 98,5\% identity) [6]. BovB (Bovine-B), a retrotransposon, is known to be the first case of horizontal transfer of genetic material between eukaryotes $[7,8,9]$. As a result of horizontal transfer, retrotransposons can have a significant impact on recipient organisms, including genetic instability, gene expression regulation and the occurrence of various disorders $[10,11]$, although the ways of horizontal transfer need to be thoroughly studied [12]. The main question is to define the vectors of horizontal transfer of retrotransposons and their association with various disorders of farm animals causing huge economic damage.

In this work, we analyzed the presence of conserved sequence identified from bovine retrotransposons recombination products in genomes of different taxa members since prokaryotes and viral sequences can be expected to act as horizontal transfer vectors.

\section{Methods}

We used for analyses the conserved sequence of retrotransposons recombination products of $266 \mathrm{bp}$ in length which we obtained earlier from 30 (LINE BovB)/(LTR/ERVK)/(LINE BovB) clusters located in 12 structural genes of bovine chromosome $1[6]$ :

CTAGCGTGTGAGATGAGTGCAATTGTGCGGTAGTTTGAGCATTCTTTGGCAT TGCCTTTCTTTGGGATTGGAATGAAAACTGACCTTTTCCAGTCCTGTGGCCACT GCTGAGTTTTCCAAATTTGCTGGCATATTGAGTGCAGCACTTTCACAGCATCAT CTTTCAGGATTTGAAATAGCTCAACTGGAATTCCATCACCTCCACTAGCTTTGTT CGTAGTGATGCTTACTAAGGCCCACTTGACTTCCATTCCAGGATGTCTGGC

The regions of similarity were identified by Basic Local Alignment Search Tool BLASTN 2.12.0+ [13]. in Nucleotide collection Database [14] with the maximum possible number of aligned sequences to display selected (5000).

\section{Results}

The BLASTN 2.12.0+ analysis of conserved sequence which is identified from bovine retrotransposons recombination products and part of BovB detected its presence with high percent identity in 43 species, including eukaryotes, prokaryotes and virus (table 1).

Table 1. Species with a high percent identity with conserved sequence derived from bovine chromosome 1 region according to BLASTN 2.12.0+ search results.

\begin{tabular}{|l|l|l|l|c|}
\hline № & \multicolumn{1}{|c|}{ Scientific name } & Common name & Taxonomy ID [15] & $\begin{array}{c}\text { Number of } \\
\text { hits }\end{array}$ \\
\hline \multicolumn{5}{|c|}{ 1. Eukaryotes } \\
\hline & 1.1 Apicomplexans & Bovine babesia & NCBI:txid189622 & 1 \\
\hline 1 & Babesia ovata & Bovine babesia & NCBI:txid5866 & 2 \\
\hline 2 & Babesia bigemina & NCBI:txid9913 & 784 \\
\hline & 1.2 Even-toed ungulates & Cattle &
\end{tabular}




\begin{tabular}{|c|c|c|c|c|}
\hline 4 & Ovis aries & Sheep & NCBI:txid9940 & 231 \\
\hline 5 & Cervus canadensis & Wapiti, American Elk & NCBI:txid1574408 & 66 \\
\hline 6 & Bubalus bubalis & Asiatic buffalo & NCBI:txid89462 & 54 \\
\hline 7 & Bos indicus $\mathrm{x}$ Bos taurus & Hybrid cattle & NCBI:txid30522 & 35 \\
\hline 8 & Cervus elaphus & Red deer & NCBI:txid9860 & 26 \\
\hline 9 & Capra hircus & Domestic goat & NCBI:txid9925 & 23 \\
\hline 10 & Oryx dammah & Scimitar-horned oryx & NCBI:txid59534 & 23 \\
\hline 11 & Bos mutus & Wild yak & NCBI:txid72004 & 22 \\
\hline 12 & $\begin{array}{l}\text { Odocoileus virginianus } \\
\text { texanus }\end{array}$ & White-tailed deer & NCBI:txid9880 & 22 \\
\hline 13 & Bison bison bison & Bison & NCBI:txid43346 & 20 \\
\hline 14 & $\begin{array}{l}\text { Ovis canadensis } \\
\text { canadensis }\end{array}$ & Bighorn sheep & NCBI:txid112262 & 19 \\
\hline 15 & Muntiacus vaginalis & Northern red muntjac & NCBI:txid9887 & 15 \\
\hline 16 & Muntiacus reevesi & Chinese muntjac & NCBI:txid9886 & 12 \\
\hline 17 & Bos indicus & Zebu & NCBI:txid9915 & 10 \\
\hline 18 & Tragelaphus spekii & $\begin{array}{l}\text { Sitatunga or } \\
\text { marshbuck }\end{array}$ & NCBI:txid69298 & 1 \\
\hline 19 & Odocoileus hemionus & Mule deer & NCBI:txid9872 & 1 \\
\hline 20 & Cervus nippon & $\begin{array}{l}\text { Sika deer or Japanese } \\
\text { deer }\end{array}$ & NCBI:txid9863 & 1 \\
\hline \multirow[t]{2}{*}{21} & $\begin{array}{l}\text { Cervus canadensis } \\
\text { canadensis }\end{array}$ & Eastern wapiti & NCBI:txid9861 & 1 \\
\hline & \multicolumn{4}{|l|}{ 1.3 Marsupials } \\
\hline \multirow[t]{2}{*}{22} & Sarcophilus harrisii & Tasmanian devil & NCBI:txid9305 & 1 \\
\hline & \multicolumn{4}{|l|}{ 1.4 Squamates } \\
\hline 23 & Podarcis muralis & Common wall lizard & NCBI:txid64176 & 4 \\
\hline 24 & Xenopeltis unicolor & Sunbeam snake & NCBI:txid196253 & 5 \\
\hline 25 & Natrix tessellata & $\begin{array}{l}\text { Checkered water } \\
\text { snake }\end{array}$ & NCBI:txid8584 & 2 \\
\hline 26 & Crotalus atrox & $\begin{array}{l}\text { Western diamondback } \\
\text { rattlesnake }\end{array}$ & NCBI:txid8730 & 1 \\
\hline 27 & Crotalus horridus & Timber rattlesnake & NCBI:txid35024 & 1 \\
\hline 28 & Naja kaouthia & Monocled cobra & NCBI:txid8649 & 1 \\
\hline 29 & Bungarus candidus & Blue krait & NCBI:txid92438 & 1 \\
\hline 30 & Ahaetulla prasina & Oriental whip snake & NCBI:txid499056 & 1 \\
\hline 31 & Enhydris enhydris & Rainbow water snake & NCBI:txid39302 & 1 \\
\hline 32 & Notechis scutatus & Mainland tiger snake & NCBI:txid8663 & 1 \\
\hline 33 & Cylindrophis ruffus & Red cylinder snake & NCBI:txid186578 & 1 \\
\hline 34 & Oligodon fasciolatus & $\begin{array}{l}\text { Small-banded Kukri } \\
\text { snake }\end{array}$ & NCBI:txid1704527 & 1 \\
\hline 35 & Homalopsis buccata & Masked water snake & NCBI:txid192599 & 1 \\
\hline 36 & $\begin{array}{l}\text { Coelognathus } \\
\text { flavolineatus }\end{array}$ & Yellow striped snake & NCBI:txid166103 & 1 \\
\hline 37 & Epicrates maurus & Brown rainbow boa & NCBI:txid1269809 & 1 \\
\hline
\end{tabular}




\begin{tabular}{|l|l|l|l|c|}
\cline { 2 - 5 } 38 & Ophiophagus hannah & King cobra & NCBI:txid8665 & 1 \\
\hline 39 & Naja siamensis & Thai spitting cobra & NCBI:txid84476 & 1 \\
\hline 40 & Python molurus & Indian rock python & NCBI:txid51750 & 1 \\
\hline & 1.5 Insects & Bed bug & NCBI:txid79782 & 1 \\
\hline 41 & Cimex lectularius & 2. Prokaryotes \\
\hline 1 & Clostridium botulinum & NCBI:txid1491 & 1 \\
\hline & \multicolumn{3}{|c|}{ 3. Viruses } & 1 \\
\hline 1 & $\begin{array}{l}\text { Jaagsiekte sheep } \\
\text { retrovirus }\end{array}$ & NCBI:txid11746 & 1399 \\
\hline
\end{tabular}

The maximum number of studied conserved sequence hits was expectedly found in eukaryotes (1397 hits), which include 1366 hits in even-toed ungulates members genome, 26 hits in squamates members genome. One hit was found in both the Tasmanian devil (Sarcophilus harrisii) genome and the Bed bug (Cimex lectularius) genome.

Hits of the studied conserved sequence were also detected in the different genomes of pathogens causing specific farm animals' diseases.

They are Babesia ovata (one hit), Babesia bigemina (two hits), Clostridium botulinum (one hit) and Jaagsiekte sheep retrovirus (one hit) with percent identity not lower than 95\% to the studied bovine conserved sequence and query coverage not lower than $65 \%$ (table 2 ).

Table 2. The comparison of conserved sequence derived from bovine chromosome 1 region with potential vectors of horizontal transfer genomes associated with various farm animal diseases.

\begin{tabular}{|l|l|l|c|c|c|c|}
\hline № & \multicolumn{1}{|c|}{$\begin{array}{c}\text { Sequence ID } \\
{[\mathbf{1 6}]}\end{array}$} & $\begin{array}{c}\text { Scientific } \\
\text { name }\end{array}$ & $\begin{array}{c}\text { Number } \\
\text { of } \\
\text { matches }\end{array}$ & $\begin{array}{c}\text { Query } \\
\text { coverage } \\
\text { (\%) }\end{array}$ & Coordinates & $\begin{array}{c}\text { Percent } \\
\text { identity } \\
\text { (\%) }\end{array}$ \\
\hline 1 & XM_029013947.1 & $\begin{array}{l}\text { Babesia } \\
\text { ovata }\end{array}$ & 1 & 100 & 402 to 668 & 99 \\
\hline 2 & LK055282.1 & $\begin{array}{l}\text { Babesia } \\
\text { bigemina }\end{array}$ & 2 & 100 & 1418 to 1683 & 95 \\
\cline { 5 - 7 } & LK054988.10 & $\begin{array}{l}\text { Babesia } \\
\text { bigemina }\end{array}$ & 1 & 86 & 2051 to 2280 & 96 \\
\hline 4 & CP027778.1 & $\begin{array}{l}\text { Clostridium } \\
\text { botulinum }\end{array}$ & 1 & 100 & 2021 to 2194 & 99 \\
\hline 5 & DQ838494.1 & $\begin{array}{l}\text { Jaagsiekte } \\
\text { sheep } \\
\text { retrovirus }\end{array}$ & 1 & 68 & 7213 to 7392 & 95 \\
\hline
\end{tabular}

\section{Discussion}

For a long time, BovB was considered to be ruminant specific but it turned out that BovB originally appeared in the reptiles' genomes, from which it was introduced in ruminant genomes by horizontal transfer $[8,9,17,18]$. Modern studies also confirm the BovB presence in marsupials $[19,20]$ and relate it to the horizontal transfer as well. In such cases bed bugs along with other blood-sucking parasites (leeches, ticks, etc.) are considered to be possible transporters of retrotransposons between species $[8,9]$. 
The identified potential vectors of retrotransposon horizontal transfer associated with various farm animal diseases are of particular interest. They are Babesia ovata, Babesia bigemina, Clostridium botulinum and Jaagsiekte sheep retrovirus.

The discovered babesia species Babesia bigemina and Babesia ovata are spread by Ixodes ticks, belong to Piroplasmida and are causative agents of babesiosis in cattle [21].

Clinical signs of babesiosis are usually characterized by fever, depression, hemolytic anemia, hemoglobinuria and jaundice, and in severe cases, the disease can lead to death. Infection caused by Babesia bigemina is characterized by a low level of parasitemia (acute hemolytic anemia, which can lead to kidney damage and organ failure), but its manifestations are more pronounced than in Babesia ovata, which is considered benign [22, $23,24]$.

The geographical distribution of pathogens is determined by the prevalence of the corresponding tick vectors. Thus, Babesia ovata is widespread in East Asian countries [25, 26,27]. In Russia Haemaphysalis longicornis, one of the tick species spreading Babesia ovata was registered in Russian Far East regions [28]. Babesiosis caused by Babesia bigemina was detected in the southern and southern east regions of Russia [29]. It should be noted that Babesia bigemina is the most closely related species to Babesia ovata based on phylogenetic and evolutionary analysis compared to other members of the babesia genus which makes it difficult to differentiate pathogens [30].

However, the contaminations of the reference genomes of Babesia ovata и Babesia bigemina by the cattle DNA could be suspected [31] as hemiparasites Babesia ovata and Babesia bigemina were cultivated in vitro using purified cattle erythrocytes [30,32]. Consequently, we are likely to provide further studies on the reference genomes obtained by more reliable methods.

Clostridium botulinum is a gram-positive rod-shaped anaerobic spore-forming motile bacterium with the ability to produce a neurotoxin known as botulism. Bacteria form spores in soil and decomposing organic material such as carcasses, bird droppings and feed stored under anaerobic conditions [33,34]. Nine different types of botulinum toxins have been identified (A, B, C, D, E, F, G, H (FA or HA), X) and more than 40 described subtypes $[35,36]$. Cattle botulism is more often associated with botulinum toxins of type $\mathrm{D} / \mathrm{C}$ and $\mathrm{C}$, but outbreaks of botulinum toxin type A diseases have been also detected [37,38]. It should be mentioned that the symptoms of botulism are also associated with different levels of susceptibility to the types of botulinum toxins, depending on the species, breed and individual. The most common symptoms observed in cattle are muscle weakness, ataxia, progressive paralysis, dysphagia [39]. It should be emphasized that the studied retrotransposons recombination products used to identify the studied conserved sequence are located in the intronic regions of structural genes closely related to the function of the central nervous system (kcne2, gart, tmem50b, il10rb, ifnar2, urb1, grik1, usp16, ltn1, cyyr1, app, jam2) [6]. Thus, the further investigation of their interaction with Clostridium botulinum neurotoxins can provide additional information about the pathogenesis of the disease.

A number of studies confirmed the role of viruses as vectors of horizontal retrotransposons transfer between eukaryotes [40]. We found the match of the region 182 bp in length of the studied conserved sequence in the Jaagsiekte sheep retrovirus (JSR) genome sequence with $94.95 \%$ identity. JSR is the causative agent of lung cancer (lung adenocarcinoma) in sheep. This disease has been described in various breeds of sheep and goats. It is believed not to affect cattle [41]. The probability of free viral particles release into colostrum or milk, when infected with the specified retrovirus, is not excluded [42].

There are studies suggesting that the presence of retroviral sequences stimulates the host's immune memory and as a consequence, protection against infection [43] For example, the insertion of babesia-like LINE L1 was found in buffalo's genome, while they 
actually demonstrate a higher level of tolerance to babesia than other species, often without any clinical signs of the disease [31].

It should be mentioned that the conserved sequence contains members of the microRNA mir-30 family [6] which are associated with muscle differentiation [44] and also involved in stress reaction and immune response development [45]. Thus, their probable regulatory role during the detected pathogens-host interactions should be thoroughly examined.

\section{Conclusions}

In this work, we identify in silico new candidate vectors of horizontal BovB retrotransposon transfer, including hemiparasites, bacterium and retrovirus. Thus, we present additional evidence that the retrotransposons distribution along the genome by horizontal transfer can significantly affect the multicellular organism regulatory system associated with protection against various diseases of farm animals. In order to improve our understanding of the biological basis of the complex pathogen-host interaction for better disease control, the role of retrotransposons in the occurrence of particular diseases of farm animals should be further thoroughly studied.

\section{References}

1. M.M. Raszek, L.L. Guan, G.S. Plastow, Frontiers in Genetics, 7, 30 (2016)

2. M. Malvisi, F. Palazzo, N. Morandi, B. Lazzari, J.L. Williams, G.Pagnacco, G. Minozzi, PLoS ONE, 11, 10 (2016)

3. P. Chuammitri, S. Srikok, D. Saipinta, S. Boonyayatra, Veterinary World, 10, 4 (2017)

4. J.N. Wells, C. Feschotte, Annu Rev Genet., 54 (2020)

5. V.I. Glazko, O.I. Skobel, G.Yu. Kosovsky, T.T. Glazko, Agricultural biology, 52, 4 (2017)

6. O.I. Skobel, V.I. Glazko, G.Yu. Kosovsky, T.T. Glazko, Izvestiya of Timiryazev Agricultural Academy, 4 (2017)

7. D. Kordis, F. Gubensek, Proc Natl Acad Sci USA, 95,18 (1998)

8. A.M. Ivancevic, R.D. Kortschak, T. Bertozzi, D.L. Adelson, Genome biology, 19, 1(2018)

9. A.M. Walsh, R.D. Kortschak, M.G. Gardner, T. Bertozzi, D.L. Adelson, Proc Natl Acad Sci USA, 110, 3 (2013)

10. F.N. Carelli, T. Hayakawa, Y. Go, H. Imai, M. Warnefors, H. Kaessmann, Genome Research, 26, 3 (2016)

11. S. Ayarpadikannan, H.S. Kim, Genomics Inform., 12, 3 (2014)

12. S.M. Dunemann, J.D. Wasmuth, Mobile DNA, 10, 24 (2019)

13. Z. Zhang, S. Schwartz, L. Wagner, W.A. Miller, J Comput Biol., 7 (2000)

14. A. Morgulis, G. Coulouris, Y. Raytselis, T.L. Madden, R.Agarwala, A.A. Schäffer, Bioinformatics, 24 (2008)

15. C.L. Schoch, S. Ciufo, M. Domrachev, C.L. Hotton, S. Kannan, R. Khovanskaya, D. Leipe, R. Mcveigh, K. O'Neill, B. Robbertse, S. Sharma, V. Soussov, J.P. Sullivan, L. Sun, S.Turner, I. Karsch-Mizrachi, Database: the journal of biological databases and curation (Oxford, 2020)

16. D. A. Benson, M. Cavanaugh, K. Clark, I. Karsch-Mizrachi, D. J. Lipman, J. Ostell, E.W. Sayers, Nucleic acids research, 41 (2013)

17. W. Puinongpo, W. Singchat, S. Petpradub, E. Kraichak, M. Nunome, N. Laopichienpong, R. Thongchum, T. Intarasorn, S. Sillapaprayoon, C. Indananda, N. Muangmai, S. Suntrarachun, S. Baicharoen, L. Chanhome, S. Peyachoknagul, K. Srikulnath, Genes, 11,11 (2020) 
18. S.A. Godakova, G.A. Sevast'yanova, S.K. Semyenova, Mol Gen Mikrobiol Virusol., 34, 1 (2016)

19. C.G. Sotero-Caio, R.N. Platt 2nd, A. Suh, D.A. Ray, Genome Biol Evol., 9, 1 (2017)

20. S. Gallus, B.M. Hallström, V. Kumar, W.G. Dodt, A. Janke, G.G. Schumann, M.A. Nilsson, Mol Biol Evol. 32, 5 (2015)

21. T.E. Onyiche, C. Răileanu, S. Fischer, C. Silaghi, Pathogens (Basel, Switzerland), 10, $2(2021)$

22. Q. Niu, Z. Liu, P. Yu, J. Yang, M.O. Abdallah, G. Guan, G. Liu, J. Luo, H. Yin, Parasites \& vectors, 8 (2015)

23. T. Sivakumar, I. Igarashi, N. Yokoyama, Veterinary Parasitology, 229 (2016)

24. G. Bohaliga, W.C. Johnson, N.S. Taus, H.E. Hussein, R.G. Bastos, C.E. Suarez, G.A. Scoles, M.W. Ueti, Parasit Vectors. 12,1 (2019)

25. T. Yoshinari, T. Sivakumar, M. Asada, B. Battsetseg, X. Huang, D.T. Lan, T. Inpankaew, A.P. Ybañez, A. Alhassan, O.M. Thekisoe, A.C. De Macedo, H. Inokuma, I. Igarashi, N. Yokoyama, J. Vet Med Sci., 75, 2 (2013)

26. T. Sivakumar, B. Tuvshintulga, A. Zhyldyz, H. Kothalawala, P.R. Yapa, R. Kanagaratnam, S. C. Vimalakumar, T. S. Abeysekera, A.S. Weerasingha, J. Yamagishi, I. Igarashi, S. Silva, N. Yokoyama, J Clin Microbiol, 56, 11 (2018)

27. L. He, R.G. Bastos, Y. Sun, G. Hua, G. Guan, J. Zhao, C.E. Suarez, Parasit Vectors, 14, $1(2021)$

28. Yu.A. Belov, T.V. Moskvina, E.M. Shchelkanov, E.G. Burukhina, Yu.G. Volkov, I.A. Popov, N.N. Kakareka, I.V. Galkina, D.V. Pankratov, A.L. Surovyi, M.Yu. Shchelkanov, Chteniya pamyati Alekseya Ivanovicha Kurencova, 30 (2019)

29. M.I. Gulyukin, V.T. Zablotskiy, W.V. Belimenko, Russian Veterinary Journal, 4 (2013)

30. J. Yamagishi, M. Asada, H. Hakimi, T. Q. Tanaka, C. Sugimoto, S.I. Kawazu, BMC genomics, 18, 1 (2017)

31. Y. Ren, C. MacPhillamy, T.H. To, T.P.L. Smith, J.L. Williams, W.Y. Low, Genomics, 113, $6(2021)$

32. A.P. Jackson, T.D. Otto, A. Darby, A. Ramaprasad, D. Xia, I.E. Echaide, M. Farber, S. Gahlot, J. Gamble, D. Gupta, Y. Gupta, L. Jackson, L. Malandrin, T.B. Malas, E. Moussa, M. Nair, A.J. Reid, M. Sanders, J. Sharma, A. Tracey, M.A. Quail, W. Weir, J.M. Wastling, N. Hall, P. Willadsen, K. Lingelbach, B. Shiels, A. Tait, M. Berriman, D.R. Allred, A. Pain. Nucleic Acids Res., 42, 11 (2014)

33. P.T. Ting, A. Freiman, Clin Med (Lond), 4, 3 (2004)

34. B.V. Eruslanov, E.A. Svetoch, I.P. Mitsevich, N.K. Fursova, I.A. Dyatlov, Bacteriology, 3, 4 (2018)

35. M.W. Peck, T.J. Smith, F. Anniballi, J.W. Austin, L. Bano, M. Bradshaw, P. Cuervo, L.W. Cheng, Y. Derman, B.G. Dorner, A. Fisher, K.K. Hill, S.R. Kalb, H. Korkeala, M. Lindström, F. Lista, C. Lúquez, C. Mazuet, M. Pirazzini, M.R. Popoff, O. Rossetto, A. Rummel, D. Sesardic, B.R. Singh, S.C. Stringer, Toxins (Basel), 9, 1 (2017)

36. S. Zhang, G. Masuyer, J. Zhang, Y. Shen, D. Lundin, L. Henriksson, S.I. Miyashita, M. Martínez-Carranza, M. Dong, P. Stenmark, Nat Commun, 8 (2017)

37. R. Souillard, D. Grosjean, T. Le Gratiet, T. Poezevara, S. Rouxel, L. Balaine, S. Macé, L. Martin, F. Anniballi, M. Chemaly, S. Le Bouquin, C. Le Maréchal, Front Microbiol., 12 (2021)

38. E.A. Frye, C. Egan, M.J. Perry, E.E. Crouch, K.E. Burbank, K.M. Kelly, J. Vet Diagn Invest., 32, 5 (2020)

39. V. Mariano, A. Nardi, S. Gradassi, P. De Santis, F. Anniballi, S. Bilei, F. Scholl, B. Auricchio, C. Bielli, M. Culicchi, G.L. Casali De Rosa, Vet Ital. 55, 1 (2019)

40. C. Gilbert, R. Cordaux, Curr Opin Virol., 25 (2017) 
41. A. Hofacre, H. Fan, Viruses, 12, 2 (2010)

42. M. Borobia, M. De Las Heras, J. Godino, L.M. Ferrer, D. Lacasta, A. Loste, J.J. Ramos, A. Ortín, J. Vet Diagn Invest (2021)

43. J.L. Hurwitz, B.G. Jones, E. Charpentier, D.L. Woodland. Viral Immunol. 30, 9 (2017)

44. Y. Zheng, K. Chen, X. Zheng, H. Li, G. Wang, Cell Stress and Chaperones, 19 (2014)

45. B.-W. Zhang, H.-F. Cai, X.-F. Wei, J.-J. Sun, X.-Y. Lan, C.-Z. Lei, F.P. Lin, X.L. Qi, M. Plath, H. Chen, International Journal of Molecular Sciences, 17, 2 (2016) 